\title{
Estudo das Propriedades Geométricas das Soluções do Problema de Roteirização de Veículos Usando Visão Computacional
}

\author{
Silvely Salomão Néia \\ Depto. Estatística, FCT, Unesp - Campus de Presidente Prudente,SP \\ silvelysalomao@fct.unesp.br \\ Almir Olivette Artero \\ Depto de Matemática e Computação Unesp - Campus de Presidente Prudente,SPl \\ almir@fct.unesp.br \\ Cláudio Barbieri da Cunha \\ POLI/Transporte/USP - São Paulo,SP \\ cbcunha@usp.br
}

\begin{abstract}
The Vehicles Routing Problem (VRP) is an NP-hard problem, and when the number of clients is very large, the task of finding the optimal solution is extremely complex. Several heuristic methods have been used to solve this problem, however, it is still necessary to find an effective way to evaluate the quality of solutions when there is no known optimal solution to the problem. This work presents a suggestion to analyze the quality of vehicle routes, based only on their geometric shapes analysis. The proposed descriptors aim to be invariants in relation to the amount of clients, vehicles and the size of the covered area. Applying the methodology proposed in this work it is possible to apply heuristic methods to obtain the route and, then, to evaluate the quality of solutions obtained. Despite being assessed problems with different configurations for the number of clients, vehicles, and service area, the results obtained with the experiments show that the proposal was useful to classify the routes on good or bad classes and obtained an accuracy rate of $99.87 \%$.
\end{abstract}

Keywords: Vehicles Routing Problem; Shape Analysis; Pattern Recognition.

\section{Introdução}

O problema de roteamento de veículos (PRV) tem aplicações importantes em diversas áreas com diferentes particularidades. O problema mais conhecido nesta classe é o Problema do Caixeiro Viajante (Traveling Salesman Problem - TSP), um problema NP-difícil, apresentado em 1934 pelo matemático Karl Menger (Garey e Johnson, 1979), que consiste em encontrar a sequência de cidades a serem visitadas por um caixeiro viajante, de modo que todas as cidades sejam visitadas exatamente uma vez e a distância total percorrida seja minimizada. Desde então, novos problemas e formulações vêm sendo propostos, com novas restrições, que incluem as capacidades dos veículos, os horários de atendimento, a duração máxima das rotas (tempo ou distância), o tamanho e a composição da frota, os tipos de veículos que podem atender determinados clientes, a precedência entre clientes, etc. Taxonomias foram apresentadas por Bodin e Golden (1981), Christofides (1985), Assad (1988) e Ronen (1988) e, mais recentemente, por Eksioglu et al. (2009), que apresentam uma classificação mais completa.

Devido a sua complexidade computacional, são muitas as propostas de resolução encontradas na literatura científica para o problema. Eksioglu et al. (2009) relatam que, como custo a ser minimizado pode ser considerado o tempo de viagem, a distância total percorrida, o número de veículos utilizados, ou o atraso. O PRV é um problema NP-difícil, deste modo, quando o número de clientes é muito grande, é muito difícil encontrar a melhor solução. Assim, vários métodos heurísticos têm sido usados para resolver este problema, no entanto, é ainda necessário encontrar um modo eficaz para avaliar a qualidade das soluções quando não existe uma solução ótima conhecida para o problema.

Este trabalho propõe uma análise da qualidade da solução a partir de um estudo das formas das rotas. As demais seções deste trabalho estão organizadas como segue. Na Seção 2 são apresentados alguns trabalhos que discutem as formas esperadas para as rotas em problemas de roteamento, além de 
alguns descritores de formas (atributos) encontrados na literatura, que são avaliados neste trabalho. Nesta seção também são apresentadas as técnicas de visualização de informações usadas durante a análise visual dos dados, e uma técnica de seleção de atributos, baseada em análise de variância. $\mathrm{Na}$ Seção 3 é apresentada a metodologia proposta neste trabalho para avaliar a qualidade das soluções para o problema do roteamento de veículos baseada na análise geométrica das formas das rotas. $\mathrm{Na}$ Seção 4 são apresentados alguns resultados usando as instâncias do tipo "A" de Augerat et al. [1998]. Por fim, na Seção 5 são apresentadas as conclusões e trabalhos futuros.

\section{Trabalhos Relacionados}

Uma tentativa inicial de explorar o formato das rotas foi feita por Gillett e Miller (1974), com uma heurística chamada Sweep Method, que sugere que as soluções ótimas tendem a apresentar uma configuração baseada em pétalas de flores. Outro trabalho apresentado por Foster e Ryan (1976), mostrou que a partir do conjunto de todas as rotas possíveis em formato de pétalas, uma solução ótima, também em formato de pétala pode ser encontrada facilmente. Uma melhoria mais significativa foi apresentada por Renaud et al. (1996), que apresentaram um algoritmo heurístico capaz de obter soluções tão boas quanto as produzidas com uma adaptação da busca Tabu, mas, com um custo computacional bem menor. Nesse sentido o formato em pétalas tem sido comum nas boas soluções. A Figura 1(a) apresenta a solução ótima para a instância A-n33-k5 (Augerat et al., 1998), onde se percebe uma estrutura em forma de pétala. Em (b) tem-se as formas das cinco rotas adotadas nesta solução.

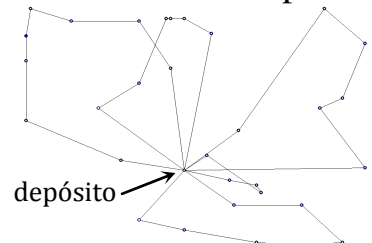

(a)

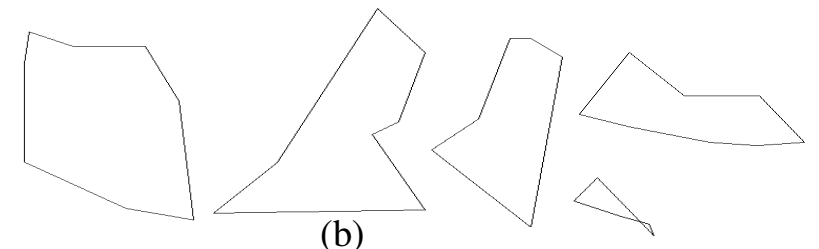

(b)

Figura 1 - a) Solução para a instância A-n33-k5; b) Formato das cinco rotas adotadas.

\subsection{Análise de Formas de Rotas}

Muitas problemas em diversas áreas podem ser resolvidos através de técnicas de visão computacional, que procuram extrair medidas a partir de uma análise de imagens (Takemura e Cesar Junior, 2002) (Costa e Cesar, 2001). Entretanto, a principal dificuldade consiste em encontrar medidas (descritores) que sejam invariantes às alterações apresentadas pelas formas, como mudanças de escala, rotações, translações e projeções. Os descritores (atributos) investigados neste trabalho para a análise das formas das rotas são (Costa e Cesar, 2001):

Custo Total - O custo total corresponde à distância total percorrida por todos os veículos. CustoMin, CustoMax e CustoMed, são as distâncias mínima, máxima e média das rotas.

Sobreposição - É a sobreposição entre as rotas pode ser um indicativo de má qualidade da solução encontrada, indicando que soluções melhores podem ser obtidas, combinando os clientes nestes trajetos.

Perímetro - O perímetro $(P)$ de uma forma é definido pela soma do comprimento de suas arestas. Embora o perímetro seja um descritor muito simples, ele é usado para obter descritores mais interessantes.

Área - A área $(A)$ de uma forma é um descritor muito simples. No caso de um contorno em uma imagem $I$, a área pode ser obtida contando todos os seus pixels contidos no interior do contorno $I_{C}$, como em (1).

$$
A=\Sigma \operatorname{pixels}(x, y) \mid(x, y) \in I_{c}
$$

Compacidade - A compacidade $(C)$ é uma medida definida pela Equação 2. A compacidade mais baixa é obtida com um círculo, que possui uma área muito grande se comparada ao seu perímetro. CompacMin, CompacMax e CompacMedia são as compacidades mínima, máxima e média das rotas. 


$$
C=\frac{P^{2}}{A}
$$

Centróide - A partir da localização do ponto central da forma, podem ser obtidos descritores como as distâncias mínimas, máximas e médias do centróide até a borda. O centróide é obtido calculando a média das coordenadas dos vértices da forma.

Diâmetro - O diâmetro $(D)$ é a maior distância entre dois pontos quaisquer da forma.

Fecho Convexo - A determinação do fecho convexo de uma forma e de sua área $\left(H_{A}\right)$ e perímetro $\left(H_{P}\right)$ são muito úteis para a caracterização das formas, sendo úteis também para a obtenção de outros descritores. O fecho convexo de uma rota é o menor polígono convexo que contém a rota.

Dimensão Fractal - A dimensão fractal $(D F)$ é um valor que descreve a irregularidade e como um objeto ocupa o espaço. A dimensão fractal de um objeto $S$ é definida pela Equação 3.

$$
D F=\lim _{r \rightarrow 0} \frac{\log \left(N_{r}\right)}{\log (1 / r)}
$$

onde $N_{r}$ é o menor número de cópias distintas de $S$ na escala $r$, que cobrem completamente o objeto $S$. Um dos métodos mais usados para se obter a dimensão fractal de um objeto em uma imagem é o Boxcounting.

Temperatura - A temperatura (T) é uma medida definida pela Equação 4, onde $H_{P}$ é o perímetro do fecho convexo. A temperatura do contorno é baseada em um formalismo da termodinâmica (DuPain et al., 1986).

$$
T=\left(\log _{2}\left(\frac{2 P}{P-H_{P}}\right)\right)^{-1}
$$

Curvatura - A curvatura é um dos mais importantes descritores que podem ser extraídos de um contorno. Dada uma curva paramétrica $c(t)=(x(t), y(t))$, a curvatura $k(t)$ é definida pela Equação 5.

$$
k(t)=\frac{x^{\prime}(t) y^{\prime \prime}(t)-x^{\prime \prime}(t) y^{\prime}(t)}{\left(x^{\prime 2}(t)+y^{\prime 2}(t)\right)^{3 / 2}}
$$

Energia de Torção (Bending Energy) - A energia de torção BE é obtida integrando o quadrado da curvatura ao longo do contorno e dividindo o resultado pelo perímetro, como na Equação 6.

$$
B E=\frac{1}{P} \int k(t)^{2} d t
$$

\subsection{Técnicas de Visualização de Informações Multidimensionais}

Por causa da grande quantidade de descritores usados neste trabalho, a técnica de visualização de informações Coordenadas Paralelas (Inselberg, 1985; Card et al., 1999) é indicada para auxiliar a análise, pois, permite visualizar todos os atributos em um mesmo gráfico 2D. Em Coordenadas Paralelas, um espaço de dimensão $n$ é mapeado em um espaço bidimensional usando $n$ eixos equidistantes e paralelos a um dos eixos principais. Cada eixo representa um atributo e, normalmente, o intervalo de valores de cada atributo é mapeado linearmente sobre o eixo correspondente. Cada item de dado é então exibido como uma linha poligonal que intercepta cada eixo no ponto correspondente ao valor do atributo associado. Cada eixo é rotulado com o nome, o menor e o maior valor de cada atributo, o que facilita a sua interpretação. Outra técnica de visualização útil para explorar dados $n$-dimensionais é o Viz3D (Artero e Oliveira, 2004), que projeta os dados em um cilindro 3D, cuja base consiste em um sistema de eixos radiais correspondente aos atributos. Os autores argumentam que as visualização obtidas com o Viz3D são similares às obtidas com uma análise de componentes principais $(P C A)$, com custo de processamento muito baixo.

\subsection{Seleção de Atributos}

Quando os dados explorados são agrupados em classes e possuem um grande número de atributos com diferentes capacidades para separar as classes, é preciso identificar os atributos mais relevantes para 
separar as classes e excluir os atributos que não possuem tanta importância na descrição do comportamento das classes, pois, estes últimos podem misturar os agrupamentos na visualização. Embora a técnica de visualização Coordenada Paralelas possa auxiliar na determinação dos atributos mais relevantes, uma técnica tradicional, que pode ser usada nesta etapa é a Análise de Variância (ANova) (Snedecor e Cochran, 1967), que compara a variância das médias dentro dos grupos (Residual) com a variância das médias entre os grupos. Quando o valor calculado de $F$ é maior que o valor crítico na distribuição de Snedecor, o atributo analisado é considerado relevante na separação das classes e, portanto, deve ser mantido na análise. A partir de uma amostra com $k$ grupos (classes), totalizando $n$ registros, determina-se o valor crítico de Snedecor $F$ pela Equação 7, sendo que valores grandes de $F$ indicam que o atributo tem maior potencial para distinguir as classes.

$$
F=\frac{n_{j} \sum\left(\bar{x}_{j}-\bar{x}\right)^{2}(n-k)}{\sum \sum\left(x_{i j}-\bar{x}_{j}\right)^{2}(k-1)}
$$

\section{3 - Análise de soluções para o PRV usando técnicas de geometria computacional}

O custo total da solução é um indicador óbvio da qualidade da rota, entretanto, apenas analisando este atributo não é possível classificar a solução como boa ou ruim, pois para problemas com vários nós (clientes) a serem atendidos é natural que o custo total seja alto, mesmo para a solução ótima, pois, o custo total depende da área coberta pelos nós. Duas possibilidades imediatas para se avaliar a qualidade de uma solução, independente da quantidade e da área coberta pelos nós seriam as razões $Q_{1}$ e $Q_{2}$, dadas nas Equações 8 e 9, que constituem dois novos atributos.

$$
\begin{aligned}
Q_{1} & =\frac{\text { CustoTotal }}{\text { ÁreaFechoConvexo }} \\
Q_{2} & =\frac{\text { Temperatura }}{\text { ÁreaFechoConvexo }}
\end{aligned}
$$

Neste trabalho, propõe-se que os descritores apresentados na Seção 2.1 e os descritores das Equações 8 e 9, possam ser aplicados para avaliar a qualidade das soluções do problema de roteamento de veículos, por meio de uma análise das formas geométricas das rotas. Estes descritores são aplicados individualmente nas rotas percorridas por cada veículo e também no trajeto total percorrido por todos os veículos, desta maneira, são previstos os descritores mínimo, máximo e média em cada solução, gerando um total de 34 atributos, que são: $a_{1}$ - CustoTotal, $a_{2}$ - CustoMin, $a_{3}$ - CustoMax, $a_{4}$-CustoMedio, $a_{5}$ - Sobreposição, $a_{6}$ - AreaMin, $a_{7}$ - AreaMax, $a_{8}$ - CompacMin, $a_{9}$ - CompacMax, $a_{10}$ - AreaMedia, a $a_{11}$-CompacMedia, $a_{12}$-BEMin, $a_{13}$-BEMax, $a_{14}$ - BEMedia, $a_{15}$ - DimFractalMin, $a_{16}$ - DimFractalMax, $a_{17}$ - DimFractalMedia, $a_{18}$ - TamanhoFechoTotal, $a_{19}$ - AreaFecho, $a_{20}$ - ComprimentoFechoMin, $a_{21}$ - ComprimentoFechoMax, $a_{22}$-AreaFechoMin, $a_{23}$ - AreaFechoMax, $a_{24}$ - ComprimentoFechoMedio, $a_{25}$ - AreaFechoMedia, a $a_{26}$ - TemperaturaTotal, $a_{27}$ - TemperMin, $a_{28}$ - TemperMax, $a_{29}$ - TemperMedia, $a_{30}$-DiametroMin, $a_{31}$ - DiametroMax, $a_{32}$ - DiametroMedio, $a_{33}-Q_{2}$ e $a_{34}-Q_{1}$.

Além destes atributos, os registros (soluções) também recebem uma classificação de acordo com o custo (soma das rotas da solução), sendo atribuída a classe 1 para as rotas boas e 2 para as ruins. A Figura 2 apresenta a solução ótima e uma solução ruim para as instâncias A-n36-k5 (pequena) e $A-n 80$ $k 10$ (grande).

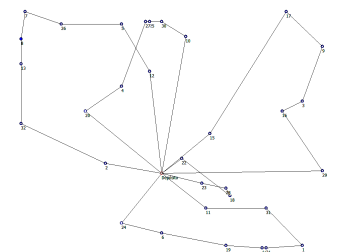

(a)

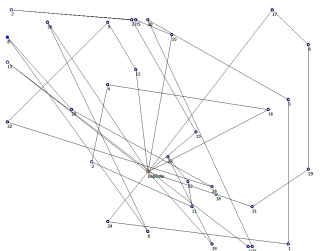

(b)

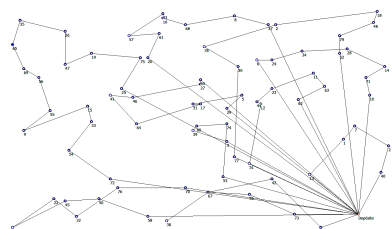

(c)

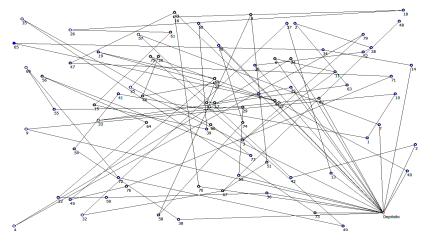

(d)

Figura 2 - a) Soluções para a instância $A-n 33-k 5$ : a) Ótima (custo total $=662.7628$ ); b) Ruim (custo total = 1,522.1065); Soluções para a instância $A-n 80-k 10$ : c) Ótima (custo total $=1,766.5000)$; d) Ruim (custo total = 4,539.7676). 
O grande desafio deste trabalho é obter descritores de forma que sejam eficazes, independentemente da quantidade de clientes, veículos e do tamanho da área atendida.

\section{4 - Experimentos}

Para investigar a utilidade destes atributos na avaliação da qualidade das soluções, independentemente do número de locais a serem visitados (clientes), do número de veículos a serem usados e das áreas de atendimento, foram usadas as instâncias: A-n32-k5, A-n33-k5, A-n33-k6, A-n34-k5, A-n36-k5, A-n37-5, A-n37-6, A-n38-k5, A-n39-k5, A-n39-k6, A-n44-k6, A-n45-k6, A-n45-k7, A-n46-k7, A-n48-k7, A-n53-k7, A-n54-k7, A-n55-k9, A-n60-k9, A-n61-k9, A-n62-k8, A-n63-k9, A-n63-k10, A-n64-k9, A-n65-k9, A-n69-k9 e A-n80-k10 (Augerat et al., 1998).

O conjunto avaliado foi construído gerando 70 soluções para cada uma destas 27 instâncias, totalizando 1.890 soluções, usando a heurística de Clarke e Wright (1964). Em seguida, foram selecionadas as 15 soluções melhores e as 15 soluções piores, resultando em 405 soluções boas (custo total baixo) e 405 ruins (custo total alto), considerado os limites máximos de capacidade dos veículos.

\subsection{Análise Visual dos Dados}

A visualização em Coordenadas Paralelas de todos os atributos é apresentada na Figura 3, onde as poligonais correspondentes às soluções boas são apresentadas em preto, enquanto que as poligonais correspondentes às soluções ruins são apresentadas em ciano.
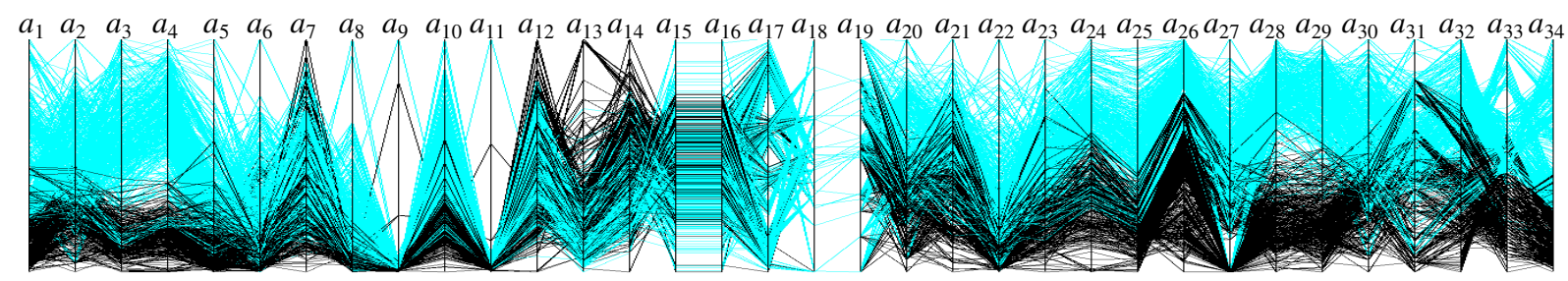

Figura 3 - Visualização em Coordenadas Paralelas dos 34 atributos (descritores).

Os valores de $F$ para estes 34 atributos são apresentados na Tabela 1.

Tabela 1 - Valores de $F$ para os 34 atributos (descritores) $a_{1}, a_{2}, \ldots, a_{34}$.

\begin{tabular}{|c|c|c|c|c|c|c|c|c|c|}
\hline$a_{1}$ & 818.0416 & $a_{8}$ & 345.9431 & $a_{15}$ & 39.6984 & $a_{22}$ & 437.7262 & $a_{29}$ & 3508.5234 \\
\hline$a_{2}$ & 671.1672 & $a_{9}$ & 7.0637 & $a_{16}$ & 39.6984 & $a_{23}$ & 857.3129 & $a_{30}$ & 666.9159 \\
\hline$a_{3}$ & 2086.1724 & $a_{10}$ & 417.7760 & $a_{17}$ & 0.1737 & $a_{24}$ & 1637.8614 & $a_{31}$ & 237.8659 \\
\hline$a_{4}$ & 3001.0991 & $a_{11}$ & 61.2329 & $a_{18}$ & $1.12 .10^{-28}$ & $a_{25}$ & 1684.3774 & $a_{32}$ & 1095.2559 \\
\hline$a_{5}$ & 352.0228 & $a_{12}$ & 56.3349 & $a_{19}$ & $1.50 .10^{-28}$ & $a_{26}$ & 1335.8672 & $a_{33}$ & 1071.1246 \\
\hline$a_{6}$ & 243.9954 & $a_{13}$ & 144.5634 & $a_{20}$ & 620.5067 & $a_{27}$ & 300.6016 & $a_{34}$ & 1204.8230 \\
\hline$a_{7}$ & 95.2011 & $a_{14}$ & 94.3906 & $a_{21}$ & 652.0983 & $a_{28}$ & 4039.3427 & & \\
\hline
\end{tabular}

Os 10 atributos com os maiores valores de $F$ de Snedecor, todos acima de 1.000, são apresentados no quadro da Figura 4(a). A visualização usando coordenadas paralelas destes 10 atributos é apresentada em (b). Nesta visualização fica clara a existência de uma separação entre as duas classes, com valores baixos na classe 1 , das soluções boas e valores altos na classe 2 , das ruins.

\begin{tabular}{|r|l|c|}
\hline \multicolumn{2}{|c|}{ Atributo } & $F$ \\
\hline$a_{28}$ & TemperMax & $4,039.3427$ \\
$a_{29}$ & TemperMedia & $3,508.5234$ \\
$a_{4}$ & CustoMedio & $3,001.0991$ \\
$a_{3}$ & CustoMax & $2,086.1724$ \\
$a_{25}$ & AreaFechoMedia & $1,684.3774$ \\
$a_{24}$ & TamanhoFechoMedio & $1,637.8614$ \\
$a_{26}$ & Temperatura Total & $1,335.8672$ \\
$a_{34}$ & CustoTotal/AreaFecho & $1,204.8230$ \\
$a_{32}$ & DiametroMedio & $1,095.2559$ \\
$a_{33}$ & temperatura/AreaFecho & $1,071.1246$ \\
\hline
\end{tabular}

(a)

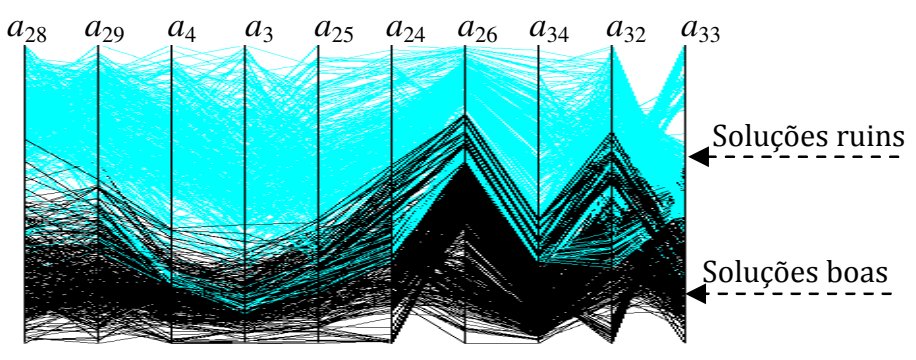

(b)

Figura 4 - Visualização em coordenadas paralelas dos registros bons (preto) e ruins (ciano), usando os 10 atributos com os maiores valores de $F$. 
Observa-se que quatro destes dez atributos são obtidos a partir da informação de temperatura, o que indica a possibilidade de este ser um atributo razoável para se distinguir soluções boas de soluções ruins para o problema do roteamento. A visualização destes registros no Viz3D é apresentada na Figura 5, onde se percebe uma separação razoável entre os marcadores nas duas classes (soluções boas em preto e soluções ruins em ciano).

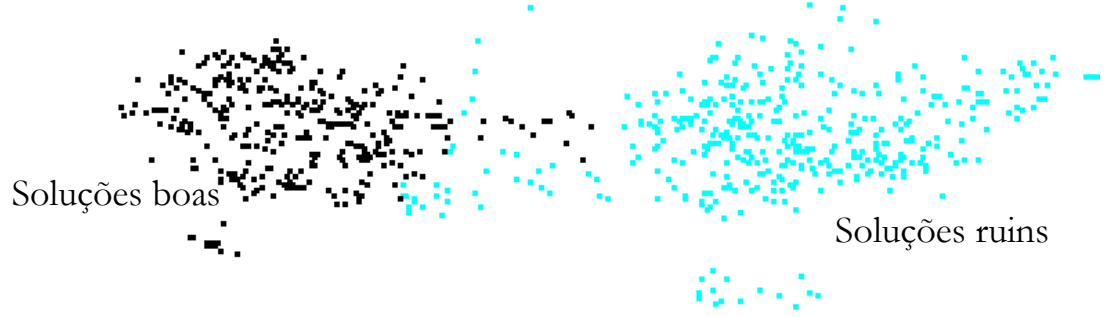

Figura 5 - Visualização Viz3D dos registros bons e ruins, usando os 10 atributos com os maiores valores de $F$.

A distribuição dos valores nas duas classes para o atributo com o maior valor $F$ (TemperMax) (Temperatura Máxima) é apresentada na Figura 6, que indica uma boa separação entre as duas classes.

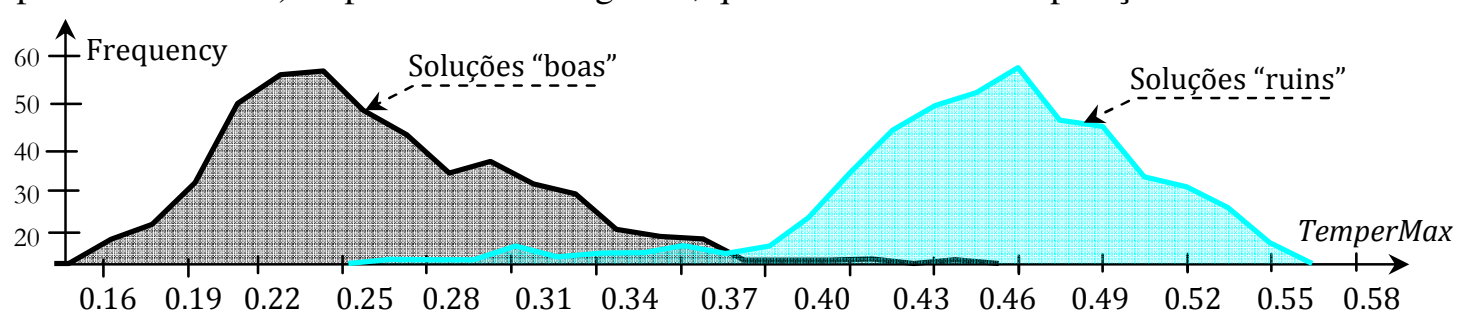

Figura 6 - Distribuição dos valores do atributo TemperMax nas duas classes "boas" e "ruins".

Como são conhecidos os custos ótimos das instâncias de Augerat et al. (1998), é possível determinar um índice de otimalidade das demais soluções obtidas para estas instâncias, dado pela relação em 10.

$$
\text { Otimalidade }=\frac{\text { CustoTotalSolução }}{\text { CustoSoluçãótima }}
$$

Neste caso, a otimalidade é igual a 1,0 para a própria solução ótima e maior que 1,0 para as demais soluções. As seis maiores correlações entre os valores de Otimalidade e os 34 atributos avaliados neste artigo são apresentadas na Tabela 2 .

Tabela 2 - Os seis atributos com as maiores correlação com a Otimalidade.

\begin{tabular}{|l|c|}
\hline Atributo & Correlação \\
\hline TemperMax & 0.944 \\
TemperMedia & 0.942 \\
AreaFechoMedia & 0.860 \\
CustoTotal/AreaFecho & 0.857 \\
TamanhoFechoMedio & 0.844 \\
TemperaturaTotal & 0.834 \\
\hline
\end{tabular}

\subsection{Análise usando uma Rede Neural Backpropagation}

Neste experimento foram usados os mesmos dados, usando uma rede neural Backpropagation (Rumelhart et al., 1986; Artero, 2009), como alternativa para avaliar a eficácia destes dez atributos com os maiores valores de $F$, na classificação das soluções nas classes "boas" e "ruins". A configuração da rede neural utilizada foi: dez neurônios na camada de entrada; cinco neurônios na camada oculta; dois neurônios na camada de saída; função de transferência logística e taxa de aprendizado 0,5 (Rumelhart et al., 1986; Artero, 2009). Após duas mil iterações de treinamento, consumindo um tempo de apenas quatro segundos, o erro máximo da rede foi de $3,19.10^{-14}$, indicando uma ótima convergência da rede, resultando em uma taxa de acerto de 99,87\% (809 acertos em 810 soluções). 


\section{5 - Conclusões}

Encontrar soluções ótimas para o problema do roteamento de veículos não é uma tarefa simples, quando existem grandes quantidades de clientes e veículos, além de diferentes tamanhos e formatos das áreas atendidas. Este trabalho propõe classificar as soluções obtidas com métodos heurísticos, em boas e ruins, a partir de uma análise baseada em descritores de formas geométricas. Além de uma classificação visual, também foi usada uma rede neural Backpropagation, que obteve uma taxa de acerto de $99,87 \%$, indicando que os atributos investigados possuem bom potencial para a avaliação das soluções, independentemente do número de clientes (variando de 32 até 80 ) e número de veículos (variando de 5 até 10). O descritor TemperMax (temperatura máxima) se destacou na discriminação das duas classes, assim como algumas variações do descritor temperatura, entretanto, muitos outros descritores ainda precisam ser avaliados em trabalhos futuros, como é o caso de descritores baseados em Fourier, Momentos, etc. (Costa e Cesar, 2001). A solução do PRV, usualmente avaliada pela distância total, pode considerar novas formas que exploram sua geometria.

\section{Referências}

1. A.O. Artero, M.C.F. Oliveira, Viz3D: Effective Exploratory Visualization of Large Multidimensional Data Sets, In. Proc. of the Computer Graphics and Image Processing Symposium, 2004, pp. 340-347, 2004.

2. A. O. Artero, Inteligência Artificial - Teórica e Prática, Editora Livraria da Física, 2009.

3. A.A. Assad, Modeling and implementation issues in vehicle routing, Amsterdam, 1988.

4. P. Augerat, J. Belenguer, E. Benavent, A. Corberán, D. Naddef, Separating capacity constraints in the CVRP using tabu search. European Journal of Operations Research, v. 106, n. 2 e 3: pp. 546 - 557, 1998.

5. L.D. Bodin, B. Golden, Classification in vehicle routing and scheduling. Networks, v. 11, n. 2, pp. 97 - 108 , 1981.

6. S.K. Card, J.D. Mackinlay, B. Shneiderman, Readings in Information Visualization, Using Vision to Think, Morgan Kaufmann, 1999.

7. G. Clarke, J.W. Wright, Scheduling of vehicles from a depot to a number of delivery points, Operations Research, 12, pp. 568-581, 1964.

8. L.F. Costa, R.M. Cesar Jr, Shape Analysis and Classification, CRC Press, Boca Raton, 2001.

9. N. Christofides, Vehicle routing, In: E.L. Lawer, J.K. Lenstra, A.H.G. Rinnooy Kan, D.B Shmoys (Eds.), The Traveling Salesman Problem: A Guided Tour of Combinatorial Optimization, NewYork, J.Wiley \& Sons, pp. 431-448, 1985.

10. Y. DuPain, T. Kamae, M. Mendès France, Can One Measure the Temperature of a Curve?, Arch. Rational Mech. Anal., 94, pp. 155-163, 1986.

11. B. Eksioglu, A.V. Vural, A. Reisman, The vehicle routing problem: A taxonomic review, Computers \& Industrial Engineering, v. 57, n. 4, pp. 1472-1483, 2009.

12. B.A. Foster, D.M. Ryan, An Integer Programming Approach to the Vehicle Scheduling Problem, Operational Research Quarterly, v. 27, 1976.

13. M.R. Garey, D.S. Johnson, Computers and Intractability: A Guide to the Theory of NP Completeness, W.H. Freeman, 1979.

14. B. Gillett, L. Miller, A Heuristic for the Vehicle Dispatching Problem. Operations Research, v. 22, pp. 340349, 1974.

15. A. Inselberg, The Plane with Parallel Coordinates, The Visual Computer, v. 1, pp. 69-92, 1985.

16. J. Renaud, F.F. Boctor, G. Laporte, An Improved Petal Heuristic for the Vehicle Routeing Problem, Journal of the Operational Research Society, v.. 47, pp. 329-336, 1996.

17. D. Ronen, Perspectives on pratical aspectos of truck routing and scheduling, European Journal of Operational Research, v. 35, n 2, pp. 137-145, 1988.

18. D.E. Rumelhart, G. E. Hinton, R. J. Williams, Learning representations by back-propagating errors, Nature v. 323 (6088), pp. $533-536,1986$.

19. G.W. Snedecor, W.G. Cochran, Statistical Methods (6th ed.), 1967.

20. C.M. Takemura, R.M. Cesar Jr, Shape Analysis and Classification using Landmarks: Polygonal Wavelet Transform, ECAI, pp. 726-730, IOS Press, 2002. 